\title{
NUEVOS RESTOS DE THEROPODA DEL JURÁSICO SUPE- RIOR-CRETÁCICO INFERIOR DE LA COMARCA DE LOS SERRANOS (VALENCIA)
}

\author{
Maite SUNEER ${ }^{1}$ Carlos de SANTISTEBAN $N^{1}$ y Àngel \\ GALOBART ${ }^{2}$ \\ ${ }^{1}$ Universitat de València. Departament de Geologia. Dr. Moliner, 50. 46100 \\ Burjassot (València). \\ ${ }^{2}$ Institut de Paleontologia "M. Crusafont”. Escola Industrial, 23. 08201 Saba- \\ dell (Barcelona).
}

Suñer, M., Santisteban, C. de \& Galobart, A. 2005. Nuevos restos de Theropoda del Jurásico Superior-Cretácico Inferior de la Comarca de los Serranos (Valencia). [ New Upper Jurassic - Lower Cretaceous Theropoda remains from 'Los Serranos' region (Valencia).] Revista Española de Paleontología, N.E. X, 93-99. ISSN 0213-6937.

\begin{abstract}
Four isolated Theropod teeth and an ungual phalanx from five new localities of Alpuente (Los Serranos, Valencia) from the Upper Jurassic - Lower Cretaceous of the Iberian Range are presented in this paper. Three teeth morphotypes are described. The results are based on size variables and denticle morphology. Three of these teeth and the phalanx are assigned to Theropoda indet. and the other tooth to Coelurosauria indet.
\end{abstract}

Keywords: Theropoda, Late Jurassic, Early Cretaceous, Iberian Range, Los Serranos, Alpuente, Valencia.

\section{RESUMEN}

Se estudian cuatro dientes aislados y una falange ungueal encontrados en cinco nuevas localidades de Alpuente (Los Serranos, Valencia) del Jurásico Superior - Cretácico Inferior del Sistema Ibérico. Se describen tres morfotipos de dientes de acuerdo con variables métricas y la morfología de los dentículos. Tres de ellos y la falange ungueal se asignan a Theropoda indet. y el cuarto diente se asigna a Coelurosauria indet.

Palabras clave: Theropoda, Jurásico Superior, Cretácico Inferior, Sistema Ibérico, Los Serranos, Alpuente, Valencia.

\section{INTRODUCCIÓN HISTÓRICA DE LA ZONA}

La comarca de Los Serranos (provincia de Valencia) es conocida en el ámbito de la paleontología por la presencia de diversos yacimientos de dinosaurios (Casanovas-Cladellas et al., 1993, 1995a,b,c, 2001). En ellos, los restos de saurópodos son relativamente abundantes, lo que contrasta con la escasez de terópodos. Hasta el momento se conoce el yacimiento de icnitas en la proximidades de la aldea de Corcolilla, el cual ha proporcionado dos morfotipos diferentes de terópodo (Santisteban et al., 2003). En cuanto a los restos directos tan sólo existen dos referencias en la bibliografía, una de Royo y Gómez (1925) en la que cita el hallazgo de dos cabezas de fémur atribuidas a 'Megalosaurus' y un trabajo publicado por Casanovas-
Cladellas et al. (1993) en el que se estudia el fragmento de un diente de carnosaurio.

El presente estudio, tiene como objetivo presentar una descripción detallada de nuevos restos aislados de terópodo encontrados, consistentes en cuatro dientes, con al menos tres morfologías y tamaños diferentes, y un fragmento de una falange ungueal.

\section{SITUACIÓN GEOGRÁFICA Y GEOLÓGICA}

Las cinco localidades en las que se han hallado los ejemplares estudiados (Fonseca, La Pacheca, La Ventura, La Cañadilla y Puntal de la Magdalena) se encuentran situadas en el término municipal de Alpuente (comarca de 


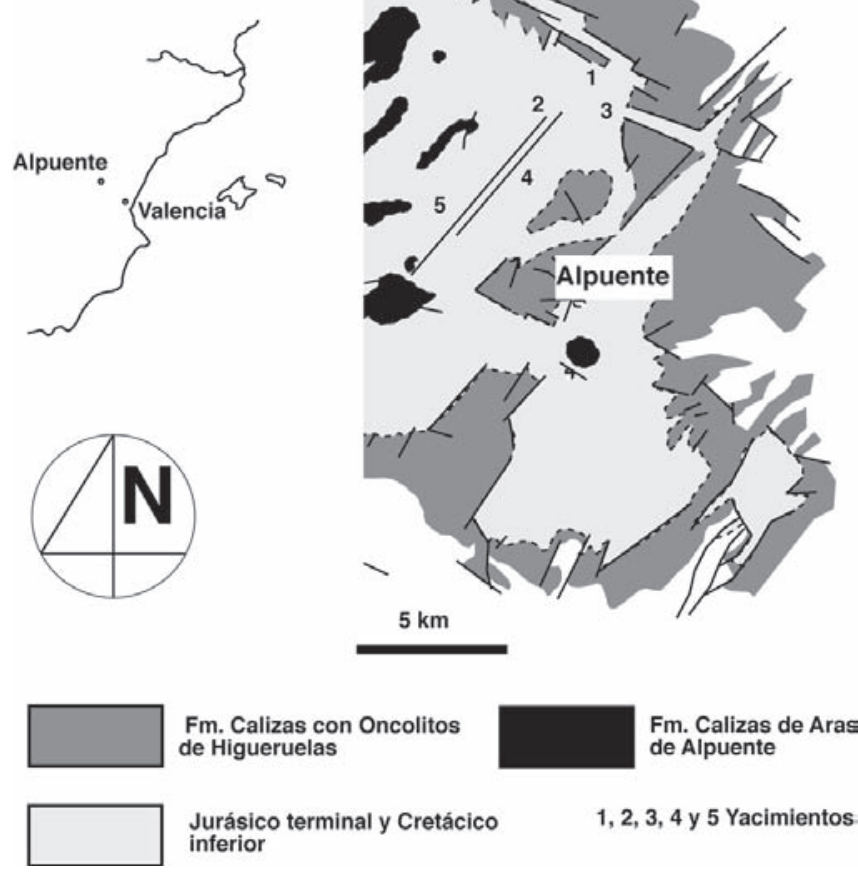

Figura 1. Situación geográfica y geológica de los yacimientos estudiados. 1) La Cañadilla, 2) Fonseca, 3) La Ventura, 4) Puntal de la Magdalena, y 5) La Pacheca. Geographic and geologic setting of the studied fossil sites. 1) La Cañadilla, 2) Fonseca, 3) La Ventura, 4) Puntal de la Magdalena, y 5) La Pacheca.

Los Serranos, Valencia) (Fig. 1). Todas las localidades se hallan en afloramientos de materiales detríticos continentales que comprenden el tránsito entre el Jurásico Superior y el Cretácico Inferior. Estos depósitos, se atribuyen a las unidades Formación Calizas, Areniscas y Arcillas de Villar del Arzobispo, Formación Calizas de la Huérguina y Formación Arenas y Arcillas del Collado, que en la zona presentan las mismas características, con una sucesión cíclica de secuencias de alta frecuencia. Estos materiales fueron sedimentados en el margen nororiental de la cuenca mesozoica Ibérica Suroccidental, en un sistema deltaico dominado por procesos de oleaje y sujeto a oscilaciones eustáticas (Santisteban, 2004)

En el registro de los materiales de los afloramientos en los que se han hallado los restos fósiles estudiados en el presente trabajo, pueden reconocerse un mínimo de cuatro secuencias deposicionales formadas, cada una de ellas, por entre cuatro y seis parasecuencias. Cada parasecuencia es un ciclo de alta frecuencia, de cuarto o quinto orden, con carácter transgresivo-regresivo. El límite entre cada secuencia deposicional es una superficie de erosión irregular, que contiene sistemas de cauces deltaicos encajados, desarrollada durante un descenso eustático. El yacimiento de la Cañadilla, se encuentra en las proximidades de la al- dea de La Canaleja, en materiales de la primera secuencia deposicional, pertenecientes a la Formación Calizas, Areniscas y Arcillas de Villar del Arzobispo. Los yacimientos de Fonseca (aldea de Corcolilla), La Ventura (aldea de La Cuevarruz) y Puntal de la Magdalena (aldea de Baldovar) se hallan en la segunda y tercera secuencias, en materiales de la Formación Calizas de la Huérguina y base de la Formación Arenas y Arcillas del Collado. El yacimiento de La Pacheca (aldea de Baldovar) se halla entre arcillas ocres con paleosuelos de la base de la cuarta secuencia, en los materiales de la parte superior de la Formación Arenas y Arcillas del Collado.

\section{INTRODUCCIÓN AL ESTUDIO DE LOS DIENTES DE TERÓPODO}

Los dientes de terópodo muestran poca variabilidad ontogenética, aunque en algunos casos la morfología dentaria varía en un mismo individuo en función de la posición que ocupe el diente en la mandíbula. Hay grupos en los que es posible distinguir los dientes premaxilares de los maxilares o dentarios y en algunos, incluso, entre los maxilares y los dentarios y entre los dentarios anteriores y los posteriores (Currie et al., 1990). No obstante, existe una relativa homogeneidad morfológica entre los dientes de diferentes terópodos, es por ello que lograr una identificación taxonómica a partir de los mismos resulte tarea difícil, especialmente en terópodos de gran tamaño. Los de pequeño tamaño sí se considera que puedan ser identificados a través de dientes aislados (Currie et al., 1990; Fiorillo \& Currie, 1994).

Por el momento, no es posible una identificación precisa de grandes terópodos del Jurásico basada en dientes aislados (Buffetaut \& Martin, 1993), por lo que la tendencia es utilizar la nomenclatura abierta.

A pesar de todo esto pueden considerarse algunas variables como son la longitud total de la corona (TCH), la longitud (FABL)y anchura (BW) de la base, la curvatura de las caras lingual y labial (Currie et al., 1990; Farlow et al., 1991) y otras basadas en la presencia o ausencia, tamaño, forma y número de dentículos (Currie et al., 1990; Buscalioni et al., 1997).

\section{DESCRIPCIÓN DIENTES}

Los ejemplares estudiados LV-1 y LC-1 se encuentran depositados en el Museo de Geología de la Universitat de València, con número de inventario MGUV-16605 y MGUV-16606, respectivamente. Los ejemplares FS-1 y LP-1 pertenecen a la colección particular de una vecina del lugar que cedió temporalmente los ejemplares para este estudio; actualmente tienen depósito provisional en el Aula de Recuperación Paleontológica de Alpuente (Diputación de Valencia). 
En las cuatro piezas dentarias se han reconocido tres Morfotipos distintos:

Morfotipo 1 (Figs. 2a, 2b y 3a)

Se halla representado por 2 dientes (FS-1 y LP-1), a los que les falta la raíz y el ápice (ver tabla para las medidas). Se caracteriza por estar comprimidos lateromedialmente, con sus caras labial y lingual convexas. En cuanto a su morfología externa, FS-1 (Fig. 2a) es simétrico, mientras que LP-1 (Fig. 2b) muestra una ligera asimetría. La presencia de dentículos en el margen posterior le da una sección basal en forma de elipse y con el margen posterior apuntado. FS-1, presenta además dentículos en ambos márgenes a partir de la mitad superior, hacia el ápice, lo que resulta en una sección transversal con los márgenes anterior y posterior apuntados (forma lenticular). El margen anterior está curvado hacia la parte posterior. En la parte basal los dentículos son pequeños y hacia el ápice son más grandes; en esta zona; la altura de cada dentículo es aproximadamente la mitad de su longitud en la base.
LP-1 es un fragmento de la base del diente donde tan sólo pueden observarse parte de los dentículos del margen posterior, los cuales están muy desgastados y no permiten hacer su descripción morfológica.

\section{Morfotipo 2 (Figs. 2c y 3b)}

Se estudia un sólo ejemplar de este morfotipo (LV-1), al cual le falta la raíz. Es un diente comprimido lateromedialmente, con sus caras labial y lingual convexas y con sección lenticular que se mantiene a lo largo de todo el diente, aunque en la base el margen anterior o mesial es más redondeado. Es de sección transversal ligeramente asimétrica, con el margen anterior curvado hacia la parte posterior. Presenta dentículos en el margen mesial y distal, en el mesial los dentículos parece que no llegan hasta la base del diente, aunque el estado de conservación del ejemplar en esta zona no nos permite observarlo con claridad. Los dentículos son uniformes en el tamaño, longitud y altura; en forma de cincel (aunque algunos de ellos tienen el borde redondeado) y se disponen perpendiculares al margen.
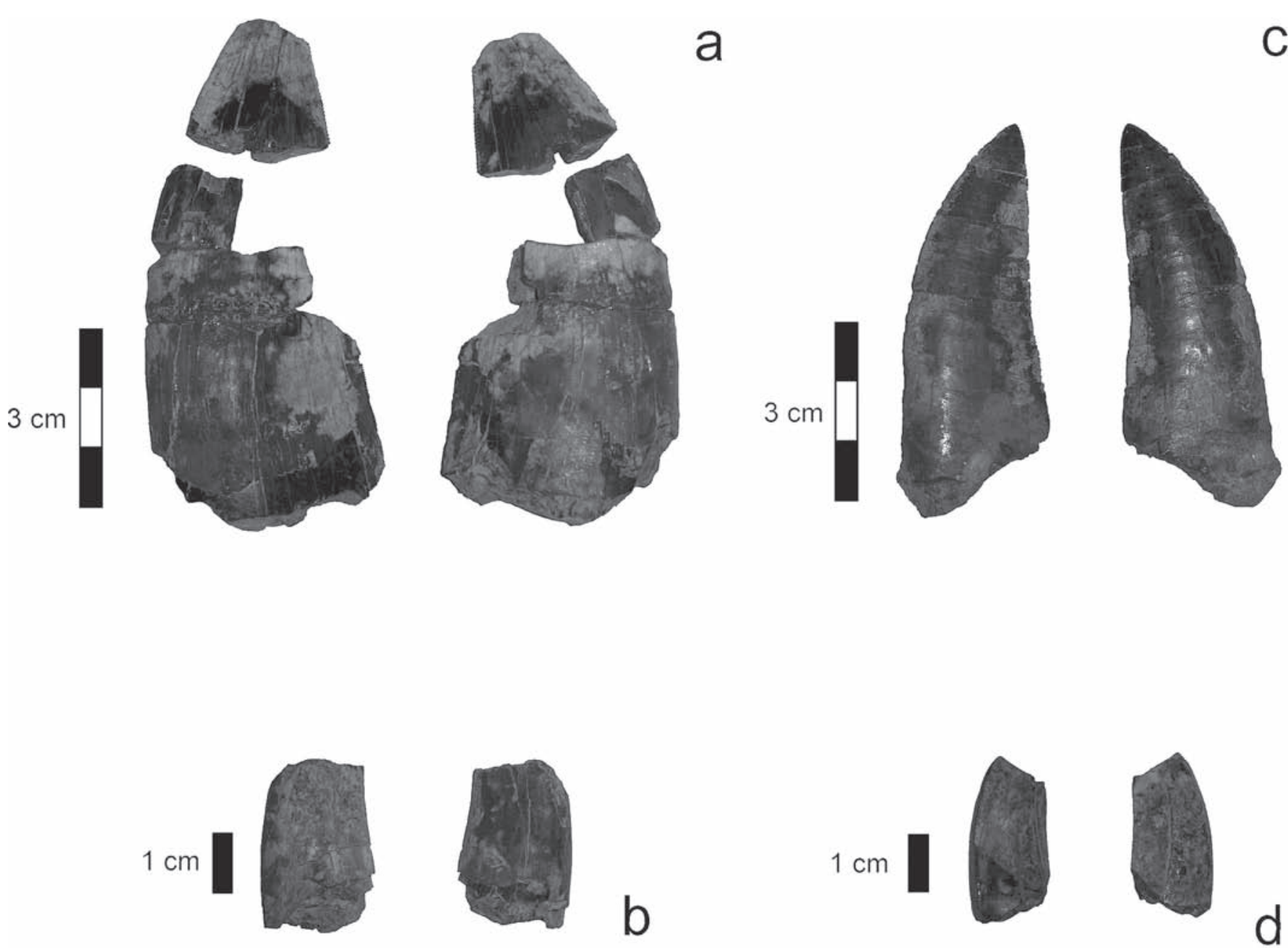

Figura 2. Vistas medial y lateral de los ejemplares FS-1 (a), LP-1 (b) (Morfotipo 1), LV-1 (c) (Morfotipo 2) y LC-1 (d) (Morfotipo 3). FS: Fonseca, LP: La Pacheca, LV: La Ventura, LC: La Cañadilla.

Lateral and medial views of the specimens FS-1 (a), LP-1 (b) (Morphotype 1), LV-1 (c) (Morphotype 2) y LC-1 (d) (Morphotype 3). FS: Fonseca, LP: La Pacheca, LV: La Ventura, LC: La Cañadilla. 


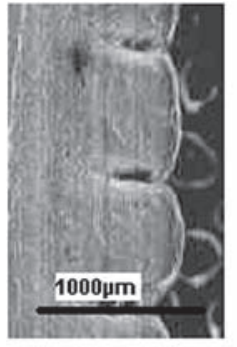

a

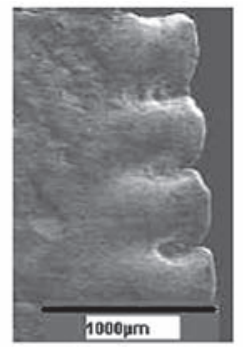

b

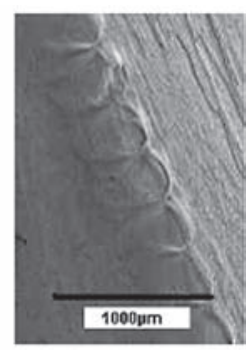

C
Figura 3. Fotografía al microscopio electrónico de barrido. a, detalle de los dentículos del Morfotipo 1(FS-1). b, detalle de los dentículos del Morfotipo 2 (LV-1). c, detalle de los dentículos del Morfotipo 3 (LC-1). SEM Photographs. a, denticles of the morphotype 1 (Specimen FS-1). b, denticles of the morphotype 2 (Specimen LV-1). c, denticles of the morphotype 3 (Specimen LC-1).

Morfotipo 3 (Figs. 2d y 3c)

Se trata de un ejemplar (LC-1) al que le falta la raíz y parte del ápice. Está comprimido lateromedialmente, con sus caras labial y lingual convexas, en sección transversal, y cóncava la cara lingual en sección longitudinal. Las carenas anterior y posterior se encuentran ambas "dirigidas" hacia la cara lingual. Es un diente asimétrico y presenta una sección cuadrangular. El margen anterior está curvado hacia el posterior y ambas caras están atravesadas longitudinalmente por una característica estría. Presenta una suave depresión en su cara lingual la cual es prácticamente paralela al margen anterior. Se observan dentículos en el margen mesial y en el distal, aunque los del margen anterior son ligeramente mayores en altura que los del posterior. Estos dentículos muestran forma de cincel y están suavemente redondeados.

\section{FALANGE UNGUEAL}

(Fig. 4)

El ejemplar estudiado se encuentra depositado en el Museo de Geología de la Universitat de València con número de inventario MGUV-15.660.

PM-1 es una pieza incompleta, a la que le falta su parte distal. Tiene una longitud de 4, $6 \mathrm{~cm}$ de longitud, pero estimamos que la longitud total del hueso sería de, al menos, $2 \mathrm{~cm}$ más; la altura es de 2,7 cm y 2,8 de anchura. Está comprimido lateromedialmente, más en su parte dorsal que ventral, lo que muestra una sección casi triangular. La simetría de esta pieza no es perfecta pues en su parte dorsal tiene una inclinación hacia la que podría ser la parte medial. Este hecho puede ser debido a que se trate de una falange del pie izquierdo o bien que la asimetría sea consecuencia de una deformación diagenética. El grado de curvatura no es excesivamente elevado y presenta una acanaladura fuertemente marcada a ambos lados y a lo largo de toda la falange, característica de los terópodos. El tubérculo flexor está suavemente marcado. La faceta de articulación es claramente cóncava y presenta una depresión central.

\section{DISCUSIÓN}

Para el estudio de los dientes, además de considerarse las variables ya comentadas, se han aplicado los criterios empleados por Buscalioni et al. (1997) para diferenciar morfotipos basados en un análisis morfológico de los dentículos. Con dicho análisis obtenemos que los ejemplares FS-1, LP-1 y LV-1 (Fig. 2a, b y c) corresponderían al morfotipo A, característico de grandes terópodos, mientras que LC-1 (Fig. 2d), presenta caracteres del morfotipo $\mathrm{B}$, característico de pequeños terópodos; sin embargo, a diferencia de éstos últimos, no presenta dentículos en forma de garfio. Este segundo morfotipo es caracterís-

\begin{tabular}{|c|c|c|c|c|}
\hline & \multicolumn{2}{|c|}{ Morfotipo 1 } & Morfotipo 2 & Morfotipo 3 \\
\hline Yacimiento & Fonseca & La Pacheca & La Ventura & La Cañadilla \\
\hline TCH (Long. Corona) & $88 \mathrm{~mm}, \mathrm{~min}$. & $27 \mathrm{~mm}, \mathrm{~min}$ & $67,5 \mathrm{~mm}$ & $28,7 \mathrm{~mm}$ min. \\
\hline FABL (Long. Base) & $37,1 \mathrm{~mm}$ & $19,4 \mathrm{~mm}$ & $24,7 \mathrm{~m}$ & $15,1 \mathrm{~mm}$ \\
\hline BW (Anchura base) & $20,6 \mathrm{~mm}$ & $15 \mathrm{~mm}$ & $11,1 \mathrm{~mm}$ & $10,2 \mathrm{~mm}$ \\
\hline Sección & Elíptica y lenticular & Eliptica & lenticular & Asimétrica \\
\hline Dentículos/2mm & $\begin{array}{c}3 \text { dent./ } 2 \mathrm{~mm} \text { y hacia } \\
\text { la base hasta 5,5 } \\
\text { dent./ } 2 \mathrm{~mm}\end{array}$ & Al menos 4dent./ $2 \mathrm{~mm}$ & 5 dent./2 mm & 5 dent./2mm \\
\hline Forma & De cincel & $?$ & De cincel & De cincel \\
\hline
\end{tabular}

Tabla 1. Medidas y otros caracteres no métricos de los ejemplares estudiados. Measurement and other no metric characters of the studied specimens. 


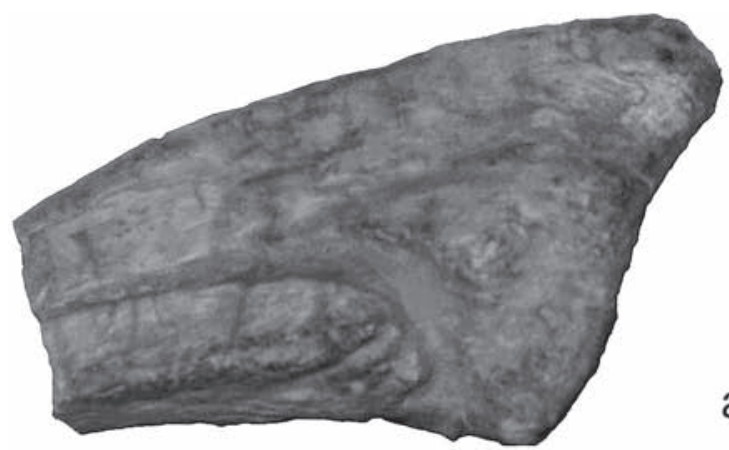

a. 1

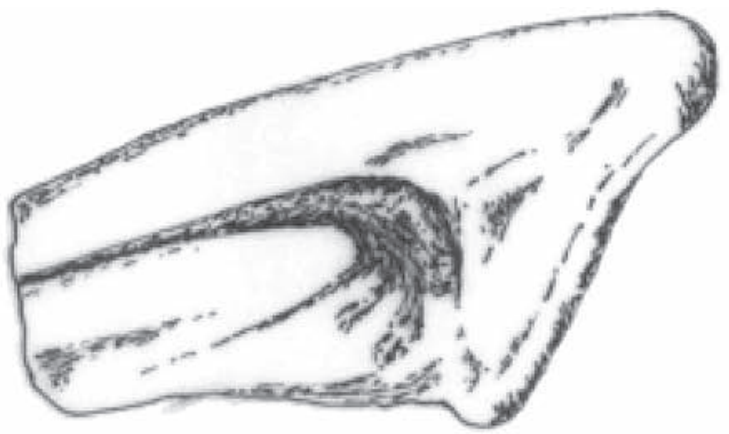

a.2

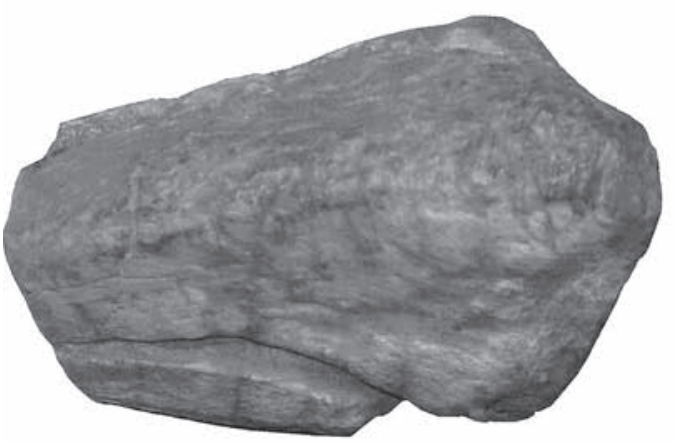

b. 1

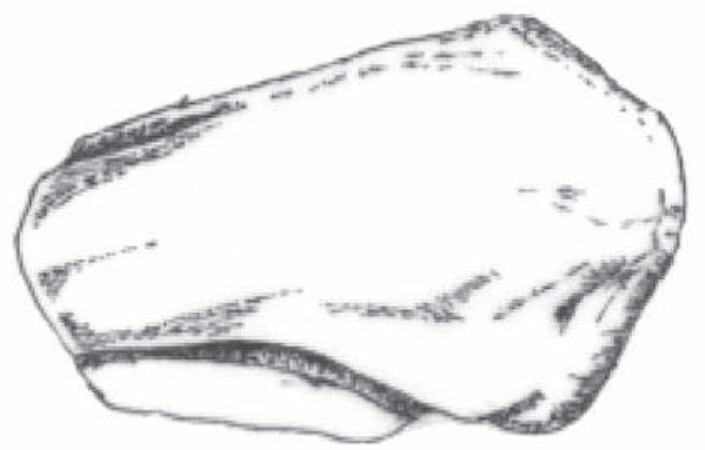

b. 2

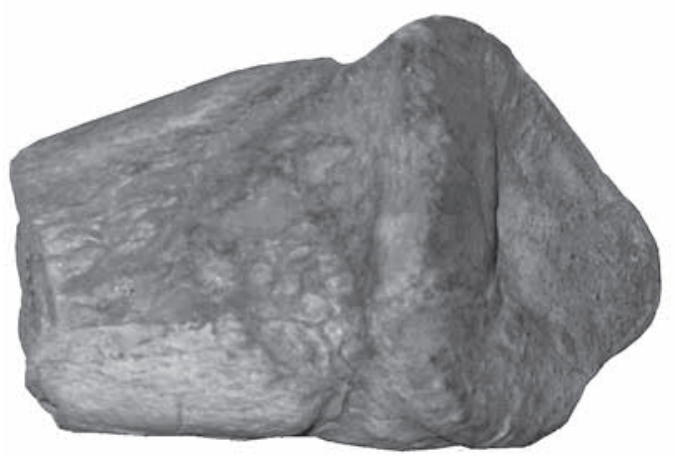

c. 1

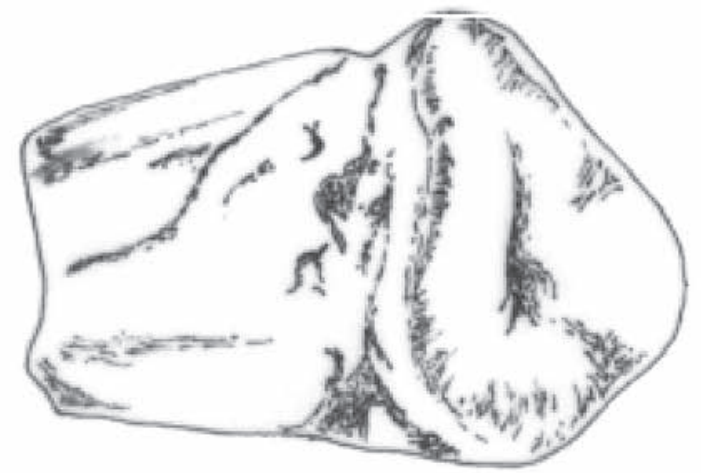

c. 2

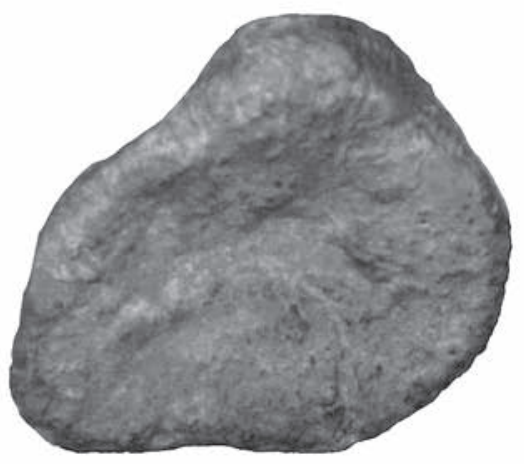

d.1

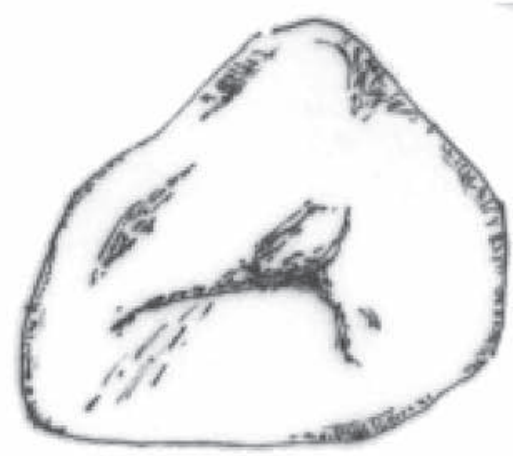

d. 2

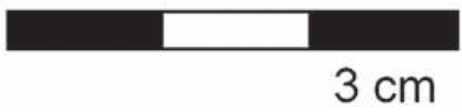

Figura 4. Vistas lateral (a.1 y a.2), dorsal (b.1 y b.2), ventral (c.1 y c.2) y proximal (d.1 y d.2) de la falange ungueal PM-1. Lateral (a.1 and a.2), dorsal (b.1 and b.2), ventral (c.1 and c.2) and proximal (d.1 and d.2) views of the ungual phalanx PM-1. 
tico de pequeños terópodos. Al igual que en algunos de los ejemplares argentinos estudiados por Buscalioni et al. (1997) este diente presenta una corona mayor de $20 \mathrm{~mm}$, los dentículos de la carena anterior están presentes, pero muestran diferencias en el tamaño respecto a los de la carena posterior.

Los ejemplares FS-1, LP-1 y LV-1 presentan las características de los dientes asignados a Megalosaurus encontrados en diferentes yacimientos datados del Jurásico terminal-Cretácico Inferior de Francia (Buffetaut \& Martin, 1993) o Tanzania (Janensch, 1925), sin embargo en la actualidad, este género ya no es considerado como válido (Allain et al., 2002), por lo que preferimos dejarlo como Theropoda indet.

El ejemplar LC-1 descartamos que se trate de un diente de espinosáurido, pues éstos presentan ambas carenas no denticuladas. El tipo de sección y la diferencia existente entre los dentículos, nos hace descartar a los grupos de grandes terópodos ceratosaurios o a tetanuros alosauroideos, o a torvosáuridos (sensu Sereno, 1997) y pensamos que puedan asignarse a Tetanurae Coelurosauria, grupo que incluye a terópodos de pequeño y gran tamaño y que aparece ya en el Jurásico Superior.

A la hora de establecer relaciones basadas en patrones de denticulación, se ha de tener en cuenta que los dentículos poseen una función relacionada con la alimentación (forma de caza y tipo de presa) y se ha de ser cauto al intentar hacer asociaciones taxonómicas, pues podemos encontrarnos con formas similares en diferentes grupos. En cuanto a la falange ungueal, ésta ha sido comparada con las del abelisáurido Ilokelesia aguadagrandensis Coria \& Salgado, 1998, Torvosaurus sp. (Galton \& Jensen, 1979; Mateus \& Antunes, 2000), Afrovenator abakensis Sereno et al., 1994, de Acrocanthosaurus atokensis Stovall \& Langston, 1950 (ver Currie \& Carpenter, 2000), Baryonyx walkeri Charig \& Milner, 1997 y Sinraptor Currie \& Zhao, 1993. Al igual que en Acrocanthosaurus el ejemplar estudiado tiene una sección transversal triangular, sin embargo este carácter consideramos que es insuficiente para asignarlo a ninguna de las familias conocidas de terópodos, si bien presenta los caracteres de una falange ungueal de gran terópodo. Así pues y por el momento se considera como Theropoda indet.

\section{CONCLUSIONES}

Los restos directos de terópodos encontrados en materiales del tránsito Jurásico-Cretácico, son, en general y especialmente en Europa y España escasos, muchos de ellos aislados y en su mayoría adscritos a unos pocos géneros o a Theropoda indet. De la misma manera, los ejemplares que estudiamos no pueden asignarse con certeza a ninguno de los taxones conocidos de terópodos. Los dientes con morfotipo 1 y 2 pertenecerían a animales de gran tamaño que por el momento consideramos como Theropoda indet. y los ejemplares con morfotipo 3 pertenecería a dinosaurios de un tamaño medio y se adscriben a Tetanurae, Coelurosauria indet.

Los dientes de terópodo encontrados en la comarca de Los Serranos proporcionan escasa información sobre las relaciones entre diferentes grupos, no obstante, resulta interesante pues hasta el momento existía un sesgo sustancial, ya que la mayor parte de restos habían sido asignados a Sauropoda o Stegosauria (Casanovas-Cladellas et al., 1995 a,b,c; 1999 y 2001). Los restos directos de terópodo hallados en materiales del Jurásico terminal/Cretácico Inferior de la Cordillera Ibérica son menos frecuentes que los de otros dinosaurios herbívoros. A los ejemplares ya conocidos (Casanovas-Cladellas et al., 1993; Barco \& Ruiz-Omeñaca, 2001a, b) se añaden con este trabajo la presencia de tres posibles taxones de terópodo del Jurásico terminal.

\section{AGRADECIMIENTOS}

Los ejemplares descritos en este trabajo, denominados FS-1 y LP-1, han sido hallados por Consuelo Villar Herrero, a quien queremos agradecer su colaboración. Así mismo deseamos agradecemos al Ayuntamiento de Alpuente las facilidades ofrecidas durante la realización de los trabajos de campo.

La versión definitiva de este trabajo se ha beneficiado de los comentarios de los dos revisores de la Revista Española de Paleontología, José Luís Sanz y Lourdes Casanovas.

El presente trabajo se ha llevado a cabo en el marco del proyecto del Ministerio de Ciencia y Tecnología BTC2001-0185C02-02. Parte del material estudiado ha sido obtenido durante la campaña de excavación autorizada y subvencionada por la Dirección General de Patrimonio de la Generalitat Valenciana, actuación con referencia 200470346-V.

\section{BIBLIOGRAFÍA}

Allain, R. \& Chure, D. J. 2002. Poekilopleuron bucklandi, the theropod dinosaur from the middle Jurassic (Barthonian) of Normando. Paleontology, 46, 1107-1121.

Barco, J.L. \& Ruiz-Omeñaca, J.I. 2001a. Primeros dientes de terópodo (Dinosauria, Saurischia) en la formación Villar del Arzobispo (Tithónico-Berriasiense): yacimientos Cuesta Lonsal y Las Cerradicas 2 (Galve, Teruel). Publicaciones del Seminario de Paleontología de Zaragoza, 5.1, 239-246.

Barco, J.L. \& Ruiz-Omeñaca, J.I. 2001b. Primeros restos postcraneales de terópodo (Dinosauria, Saurischia) en la Formación Villar del Arzobispo (Tithónico-Berriasiense): Un centro vertebral caudal del yacimiento Carretera (Galve, Teruel). Publicaciones del Seminario de Paleontología de Zaragoza, 5.1, 247-254.

Buffetaut, E. \& Martin, M. 1993. Late Jurassic dinosaurs 
from the Boulonnais (Northern France): a review. Revue de Paléobiologie, Vol. especial 7, 17-28.

Buscalioni, A.D., Gasparini, Z., Pérez-Moreno, B.P. \& Sanz, J.L. 1997. Argentinian theropods: first morphological analysis on isolated teeth. I European Workshop on Vertebrate Paleontology. Geological Society of Denmark, On line series, 1.

Canudo J.I. \& Ruiz-Omeñaca, J.I. , 2003. Los restos directos de dinosaurios terópodos (excluyendo aves) en España. En: Dinosaurios y otros Reptiles Mesozoicos en España (coor. F. Pérez Lorente). Instituto de Estudios Riojanos, Ciencias de La Tierra, 26, Logroño, 347- 374.

Casanovas-Cladellas, M.L., Santafe-Llopis, J.V. \& Santisteban-Bové, C. 1993. First dinosaur teeth from the Lower Cretaceous of Benicatazara (Aras de Alpuente, Valencia. Revue de Paléobiologie, Vol. especial 7, 37-44.

Casanovas-Cladellas, M. L., Santafé-Llopis, J. V., PeredaSuberbiola, X. \& Santisteban-Bové, C. 1995 a. Presencia, por primera vez en España, de dinosaurios estegosaurios (Cretácico Inferior de Aldea de Losilla, Valencia). Revista Española de Paleontología, 10, 83-89.

Casanovas-Cladellas, M. L., Santafé-Llopis, J. V. \& PeredaSuberbiola, X. 1995 b. Nuevo material de estegosaurios en el Cretácico Inferior de Valencia. Paleontologia $i$ Evolució, 28-29, 269-274.

Casanovas-Cladellas, M. L., Santafé-Llopis, J. V. \& Santisteban-Bové, C. 1995 c. Dacentrurus armatus (Stegosauria, Dinosauria) del Cretácico Inferior de Los Serranos (Valencia, España). Revista Española de Paleontología, 10, 273-283.

Casanovas-Cladellas, M. L., Santafé-Llopis, J. V., de Santisteban-Bové, C. \& Pereda-Suberbiola, X. 1999. Estegosaurios (Dinosauria) del Jurásico superior-Cretácico inferior de la comarca de Los Serranos. Revista Española de Paleontología, $\mathbf{n}^{\circ}$ Homenaje al Prof. J. Truyols, 57-63.

Casanovas-Cladellas, M.L., Santafe, J.V. \& Sanz, J.L. 2001. Losillasaurus giganteus, un nuevo saurópodo del tránsito Jurásico-Cretácico de la cuenca de "Los Serranos" (Valencia, España). Paleontologia $i$ Evolució, 32-33, 99-122.

Charig, A.J. \& Milner, A.C. 1997. Baryonix walkeri, a fisheating dinosaur from the Wealden of Surrey. Bulletin of the Natural History Museum of London (Geology), 53, 11-70.

Coria, R.A. \& Salgado, L. 1998. A basal Abelisauria NOVAS, 1992 (Theropoda-Ceratosauria) from the Cretaceous of Patagonia, Argentina. GAIA, 15, 89-102.

Currie, P.J. \& Carpenter, K. 2000. A new specimen of Acrocanthosaurus atokensis (Theropoda, Dinosauria) from the Loweer Cretaceous Antlers Formation (Lower Cretaceous, Aptian) of Oklahoma, USA. Geodiversitas, 22, 207-242.
Currie, P.J. \& Zhao, X.J. 1993. A new carnosaur (Dinosauria, Theropoda) from the Jurassic of Xinjiang, People's Republic of China. Canadian Journal of Earth Science, 30, 2037-2081.

Currie, P.J., Rigby , J.K. \& Sloan, R.E. 1990. Theropod teeth from the Judith River Formation of southern Alberta, Canada. In: Dinosaurs perspectives and Approaches (Ed. K. Carpenter \& P.J. Currie). Cambridge University Press, New York, 107-125.

Farlow, J.O., Brinkman, D.L., Abler, W.L. \& Currie, P.J. 1991. Shize, shape and serration density of theropod dinosaur lateral teeth. Modern Geology, 16, 161-198.

Fiorillo, A. \& Currie, P. 1994. Theropod teeth from the Judith River Formation (Upper Cretaceous) of SouthCentral Montana. Journal of Vertebrate Paleontology, 14, 74-80.

Galton P.M. \& Jensen, J.A. 1979. A newlarge theropod from the Upper Jurassic of Colorado. Brigham Young University Geology Studies, 26 (2), 1-12.

Janensch, W. 1925. Die Coelurosaurier und Theopoden der Tendaguru-Schichten Deutsch-Ostafrikas. Paleontographica (Suppl.7), 1, 1-29.

Mateus, O. \& Antunes, M.T. 2000. Torvosaurus sp. (Dinosauria: Theropoda) in the Late Jurassic of Portugal. Libro de Resúmenes del I Congreso Ibérico de Paleontología, 115-117.

Royo y Gómez, J. 1925. Sobre los restos de reptiles weáldicos de Benagéber (Valencia) y algunos moluscos cuaternarios de Villavieja (Castellón). Acta del Boletín de la Real Sociedad Española de Historia Natural, 25, 252.

Santisteban, C. 2004. Ambiente sedimentario y ciclicidad estratigráfica de los materiales de la Formación "Arenas y arcillas del Collado" (Cretácico inferior), en el sector de Alpuente (Valencia). Geogaceta, 35, 15-18

Santisteban, C., Gaete, R., Galobart, A. \& Suñer, M. 2003. Rastros de dinosaurios en el Jurásico terminal (Facies Purbeck) de Corcolilla (Los Serranos, Valencia). En: Dinosaurios y otros Reptiles Mesozoicos en España (Coor. F. Pérez Lorente). Instituto de Estudios Riojanos, Ciencias de La Tierra, 26, Logroño, 33-40.

Sereno, P.C. 1997. The origin and evolution of dinosaurs. Annual Reviews of Earth Planet Sciences, 25, 435-489.

Sereno, P.C., Wilson, J.A., Larsson, H.C.E., Dutheil, D.B. \& Sues, H.D. 1994. Early Cretaceous Dinosaurs from the Sahara. Science, 266, 267-270.

Stovall, J.W. \& Langston, W. Jr. 1950. Acrocanthosaurus atokensis, anew genus and species of Lower Cretaceous theropod from Oklahoma. The American Midland Naturalist, 43, 696-728.

Manuscrito recibido: 12 de Enero, 2004 Manuscrito aceptado: 30 de Mayo, 2005 